Proc Indian Natn Sci Acad $\mathbf{8 1}$ No. 1 February 2015 Special Issue, pp. 282-287

(c) Printed in India.

DOI: $10.16943 / \mathrm{ptinsa} / 2015 / \mathrm{v} 81 \mathrm{i} 1 / 48083$

\title{
Accretion Dynamics in SdS Space
}

\author{
SONALI SAHA* \\ ${ }^{1}$ Department of Physics, Sarojini Naidu College for Women, Kolkata 700 028, India
}

(Received on 30 April 2014; Accepted on 09 August 2014)

Generally a centrally located accreting super massive $\left(\sim 10^{8} M_{\odot}\right)$ black hole is thought to be playing the role of the gravitational powerhouse of gigantic scale in AGN. The accretion process for such a black hole may be studied using general relativistic as well as post Newtonian scheme for various possible flow geometries with different equation of states of the accreted matter. For the sake of simplicity, hydro-dynamical flow models are generally adopted to get an analytical estimate for those accretion process. In our work, accretion process over a region far beyond the dimension of a single galaxy by a much more massive object ( $\sim 10^{18} M_{\odot}$, a cluster of galaxies or a possible super-giant black hole) has been studied where the large scale feature of the space-time structure of the Universe may be relevant. In the non-rotating limit of the accreting black-hole with accelerated expansion of the Universe, Schwarzschild-de Sitter (SdS) space is chosen to be a suitable one. Pseudo-Newtonian potential describing the gravitational field of static and spherically symmetric black holes in the Universe with a repulsive cosmological constant, i.e. for SdS space, has been recently introduced by Stuchlik and Kovar. Using this potential, here we have studied the phase topology of the flow and also the possibility of formation of stationary shocks both in adiabatic and isothermal limits in accretion discs. In AGN, the existence of stationary shock is believed to be a plausible explanation of the extremely hot annular region there. In our study, in adiabatic (and in isothermal) cases, a significant region of parameter space allow shock formation and those regions shift in comparison with the same for Schwarzschild case due to the effect of cosmological constant in SdS space.

\section{Key Words : Accretion; Black Hole; Comological Constant; Shock}

\section{Introduction}

Accretion flow surrounding a black hole was the subject of many theoretical studies in Astrophysics for last half a century or so (see Frank et al., 2002). Particularly after emergence of AGN model, where black hole

*Author for Correspondence : E-mail: snl_saha@yahoo.com 
accretion is supposed to be the central power house, as the unifying scheme (Frank et al., 2002) to explain the behaviour of exotic astronomical objects like Seyfart galaxies, Quasars, Blazars etc. According to this idea accretion dynamics around black hole should bear the signature of the surrounding space-time structure and hence properties of the black hole itself.

Schwarzschild de Sitter (SdS) space-time with positive cosmological constant may be considered to be a plausible model of accelerating Universe with a centrally located spherical mass distribution (Rindler, 2006). Hence it is expected that for a sufficiently massive black hole, accretion dynamics over a large length scale should bear the signature of SdS space and hence the effect of the cosmological constant at large length scale too.

The accretion dynamics in general relativistic (GR) set up is quite complex to handle analytically. An alternative approach (see e.g. Nag et al., 2012; Bilic et al., 2014) is to handle the problem in Newtonian framework with an effective potential (pseudo-potential) which may mimic the general relativistic motion at least in a region much away from the event horizon. For $\mathrm{SdS}$ space such pseudo potential has recently been prescribed (Stuchlik and Kovar, 2008) in literature.

In this work using the prescribed potential (Stuchlik and Kovar, 2008) we have explored the accretion dynamics around a centrally located non-rotating black hole in the accelerating Universe model and compared it with the same in the non-accelerating one. In the present context, the cosmological horizon is obviously far away from the region where accretion flow takes place but the signature of the non-zero cosmological constant remains present, however small, in the space-time curvature of the accretion region. We have studied the accretion flow dynamics for low angular momentum limit considering conical accretion disc geometry for both adiabatic and isothermal cases. We have investigated the possibility of shock formation in the accretion disc using the shock conditions in the respective cases and also the effect of the cosmological constant, hence of the accelerating nature of the Universe on the allowed region for the shock formation in the parameter space.

\section{Accretion Process}

In case of axisymmetric, inviscid steady flow, the equations which govern the drift along the radial direction are the Euler's equation (radial component)

$$
v \frac{d v}{d r}+\frac{1}{\rho} \frac{d P}{d r}+\phi^{\prime}(r)-\frac{\lambda^{2}}{r^{3}}=0
$$

and the continuity equation

$$
\frac{d}{d r}(\rho v r H)=0
$$


where $\phi^{\prime}(r)$ is the spatial derivative of of the generalised pseudo-Newtonian potential $\phi(r), \lambda$ is the conserved specific angular momentum of the flow, $P$ is the pressure of the flowing gas with density $\rho, H$ is the local thickness of the disc. In this study we have used both polytropic $\left(P \propto \rho^{\gamma}\right)$ and isothermal $(P \propto \rho)$ prescriptions of the equation of state for conical flow (where $H=A r, A$ being the proportionality constant). The bulk radial velocity $v$ has been scaled by the local speed of the sound $c_{s}=\left(\frac{\partial P}{\partial \rho}\right)^{1 / 2}$.

\section{Adiabatic Limit}

Using equations (1) and (2) after integrating and rearranging the terms we get two critical point conditions as

$$
v_{c}^{2}=c_{s c}^{2}=\left[r_{c} \phi^{\prime}(r)-\frac{\lambda^{2}}{r_{c}^{2}}\right]\left[1+r_{c} \frac{H^{\prime}\left(r_{c}\right)}{H\left(r_{c}\right)}\right]^{-1},
$$

(where the subscript $c$ indicates the value at critical point) and

$$
\frac{1}{2}\left(\frac{\gamma+1}{\gamma-1}\right)\left[r_{c} \phi^{\prime}\left(r_{c}\right)-\frac{\lambda^{2}}{r_{c}^{2}}\right]\left[1+r_{c} \frac{H^{\prime \prime}\left(r_{c}\right)}{H\left(r_{c}\right)}\right]^{-1}+\phi\left(r_{c}\right)+\frac{\lambda^{2}}{2 r_{c}^{2}}=\mathcal{E}
$$

$\mathcal{E}$ being the constant of integration interpreted as specific energy. These equations are used to find the critical points.

\section{Isothermal Limit}

Using the linear expression for $P$ for isothermal case, the first integral of equation (1) becomes

$$
\frac{v^{2}}{2}+c_{s}^{2} \ln \rho+\phi(\mathrm{r})+\frac{\lambda^{2}}{2 \mathrm{r}^{2}}=\mathcal{C}
$$

where $\mathcal{C}$ is a constant of integration which can be found out using the fact that at large distance flow solution decays to zero and $\mathcal{C}=c_{s}^{2} \ln \rho_{\infty}$. In isothermal case also, one gets the critical point condition as $v_{c}=c_{s}$. But in this case the sound speed is constant (Frank et al., 2002) which can be expressed as $c_{s}=\Theta T^{1 / 2}$ where $\Theta=\left(\kappa / \mu m_{H}\right)^{1 / 2}$ and where $m_{H}=$ proton mass, $\mu=$ mean molecular weight, $\kappa=$ Boltzman constant. For conical disc geometry one gets the critical points using the relation,

$$
r_{c} \phi^{\prime}\left(r_{c}\right)-\frac{\lambda^{2}}{r_{c}^{2}}=2 c_{s}^{2}
$$

\section{Phase Portrait of Stationary Flow}

To draw the phase portrait of the stationary flow, one has to identify the physically tenable solutions. At large distance from the black hole the accretion flow is subsonic and it is supersonic very close to the accretor due to the presence of event horizon. Thus the accretion process shows transonic behaviour. This transonic accretion solution is realised mathematically as the critical solution, in the phase portrait of local radial 
Mach number $\left(\mathcal{M}=v / c_{s}\right)$ and radial distance $(r)$, outside the event horizon. For low angular momentum axisymmetric black hole accretion there may exist more than one critical solutions in the phase portrait (see Fig. 1). Then the accretion process consequently becomes multicritical. In astrophysics, this multicritical flow dynamics is of special interest as it may allow shock formation in low angular momentum axisymmetric black hole accretion under certain conditions (see the next section).

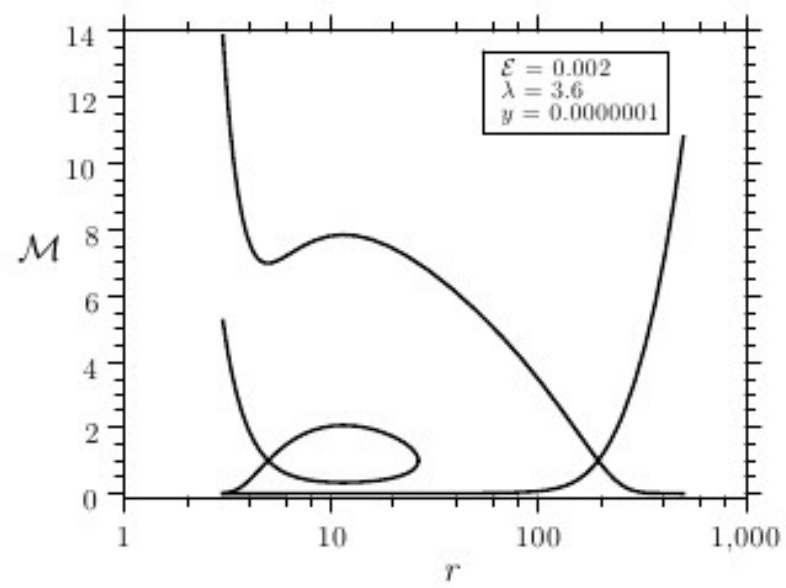

Fig. 1: Typical phase portrait of the flow

\section{Parameter Space Study of the Stationary Solutions}

In this work, the recently proposed SdS pseudo-potential (Stuchlik and Kovar, 2008), has been used to study the signature of accelerating universe on accretion flow and shock formation, where $y=\Lambda \frac{M^{2}}{3}$ is cosmological parameter, $M$ is the black hole mass and $\Lambda$ is the cosmological constant in the SdS metric.

$$
\phi(r)=\frac{r^{3} y-3 r y^{\frac{1}{3}}+2}{2\left(1-3 y^{\frac{1}{3}}\right)\left(2-r+r^{3} y\right)}
$$

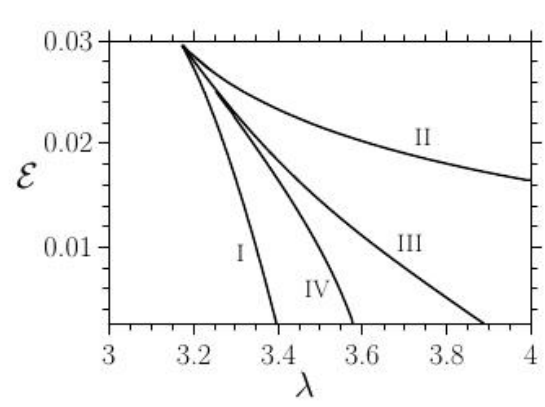

A: Adiabatic flow

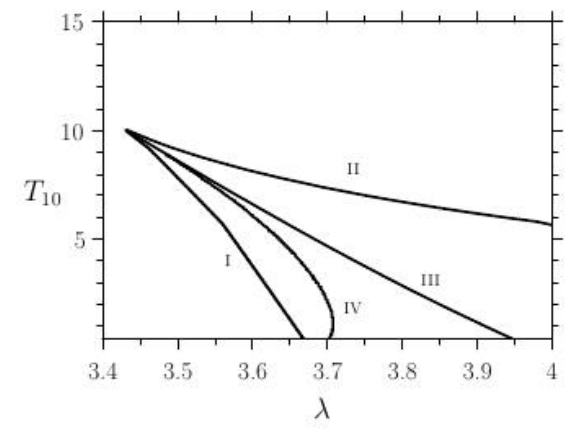

B: Isothermal flow

Fig. 2: Flow parameter space for different flow conditions. See text for explanations 


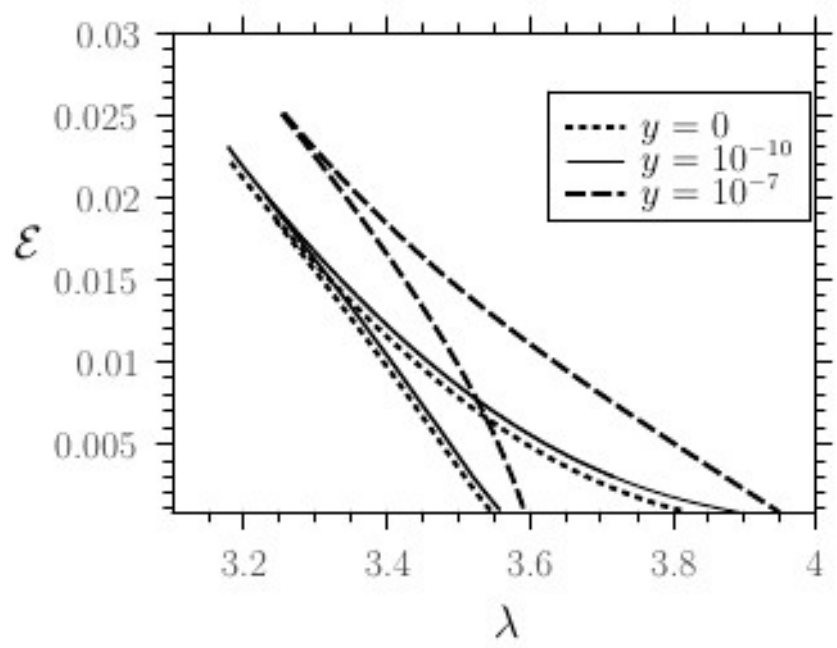

Fig. 3: Variation of shock region with that of parameters

For super massive black hole $\left(M \approx 10^{11} M_{\odot}\right), y \sim 10^{-24}$ (Stuchlik and Hledik, 1999). It is to be metioned here that, using the pseudo potential approach, one can replace the space time curvature by an equivalent potential in the background of a flat space time, where the curvature is not too high. We have studied the effect of the variation of the parameter $y$ on accretion dynamics, particularly in conical disc geometry in case of non rotating black hole and in low angular momentum limit of the accretion flow where the effect of viscosity is negligible. Fig. 2A shows the variation of the number of critical points for polytropic flows $(\gamma=4 / 3)$ with the variation of $\mathcal{E}$ and $\lambda$ for $y=10^{-7}$. The wedge shaped region bounded by I and II depicts the parameter values corresponding to the three critical points in the constant energy flow solutions for conical disc geometry. Outside this region, there exist only one critical point. Within the region I and II, there is a subregion bounded by IV and III where at some distance, outside the event horizon, the condition for shock formation (Chakrabarti and Das, 2001)

$$
\frac{[\gamma \mathcal{M}+(1 / \mathcal{M})]^{2}}{2+(\gamma-1) \mathcal{M}^{2}}=\text { constant }
$$

is being satisfied in addition to the condition of higher entropy at inner critical point in comparison with that at outer one. This wedge shaped subregion in parameter space is named as shock region. The result for the same study for the isothermal case is shown in Fig. $2 \mathrm{~B}$ (with shock condition $\mathcal{M}+1 / \mathcal{M}=$ a constant) for the same value of $y$ as chosen for adiabatic study ( $T_{10}$ being the flow temperature in the unit of $10^{10}$ ${ }^{o} K$ ). It is clear from the above figures that for lower values of $\mathcal{E}$ (and $T$ ) for polytropic flow (and isothermal flow) there is an interval of $\lambda$ where shock formation is allowed and this interval shrinks for higher values of $\mathcal{E}($ and $T)$. The effect of variation of $y$ in shock region which is of primary interest for this work is shown in Fig. 3 for three different values of $y$. It is evident from the figure that the effect of $y$ is only slightly 
appreciable for $y=10^{-10}$ which corresponds to $M \approx 10^{18} M_{\odot}$ and hence it is almost non-existent for the super massive black hole $\left(M \approx 10^{11} M_{\odot}\right.$ and $y=10^{-24}$ ) accretion process; whereas for $y=10^{-7}$ (which corresponds to $M \gtrsim 10^{19} M_{\odot}$ ) there is a significant shift in shock region towards higher $\lambda$ and $\mathcal{E}$.

In conclusion there is a sizeable region in parameter space which allows shock formation for black hole accretion in SdS space irrespective of the black hole mass. But the effect of cosmological constant is not appreciable even in the case of super massive black hole $\left(\lesssim 10^{11} M_{\odot}\right)$. For such an effect to be appreciable, there must exist, if possible, some super giant black hole $\left(M \gtrsim 10^{18} M_{\odot}\right)$.

\section{Acknowledgement}

This work is only a part of the larger work being done in collaboration with Tapas K. Das, HRI, Allahabad, India and others. The author likes to acknowledge him and also Sourav Bhattacharya of the same institute for various useful discussions regarding the present work.

\section{References}

1. Bilic N, Choudhary A, Das TK and Nag S (2014) An analytical study on the multi-critical behaviour and related bifurcation phenomena for relativistic black hole accretion. Class Quantum Grav 31035002

2. Chakrabarti SK and Das S (2001) Model dependence of transonic properties of accretion flows around black holes. MNRAS 327808

3. Frank J, King A and Raine D (2002) Accretion Power in Astrophysics 3rd Edition, Cambridge University Press

4. Rindler W (2006) Relativity 2nd Edition, Oxford University Press

5. Nag S, Acharya S, Ray AK and Das TK (2012) The role of flow geometry in influencing the stability criteria for low angular momentum axisymmetric black hole accretion. New Astronomy 17285

6. Stuchlik Z and Kovar J (2008) Pseudo-Newtonian Gravitational Potential for Schwarzschild-De Sitter SpaceTimes. Int J Mod Phys D17 2089

7. Stuchlik Z and Hledik S (1999) Some properties of the Schwarzschild-de Sitter and Schwarzschild-anti-de Sitter spacetimes. Phys Rev D60 044006. 